\title{
REMARKS ON THE WELL-POSEDNESS OF THE EULER EQUATIONS IN THE TRIEBEL-LIZORKIN SPACES
}

\author{
ZIHUA GUO AND KUIJIE LI*
}

\begin{abstract}
We prove the continuous dependence of the solution maps for the Euler equations in the (critical) Triebel-Lizorkin spaces, which was not shown in the previous works $[6,7,9]$. The proof relies on the classical Bona-Smith method as [12], where similar result was obtained in critical Besov spaces $B_{\infty, 1}^{1}$.
\end{abstract}

\section{INTRODUCTION}

This article addresses the ideal incompressible Euler equations in $\mathbb{R}^{d}, d \geq 2$ :

$$
\partial_{t} u+u \cdot \nabla u+\nabla p=0, \quad \nabla \cdot u=0, \quad u(0, x)=u_{0}(x),
$$

where $u: \mathbb{R}^{+} \times \mathbb{R}^{d} \rightarrow \mathbb{R}^{d}$ represents the velocity vector, $p$ is scalar pressure, $u_{0}$ is the initial condition verifying $\nabla \cdot u_{0}=0$.

There are extensive literatures on the mathematical analysis of the Euler equations. The Cauchy problem in very general functional setting has been well studied. Kato [15] constructed a unique local in time regular solution to the 3D Euler equation with initial data in $H^{m}\left(\mathbb{R}^{3}\right), m \geq 3$. Similar result was obtained for initial data belonging to $H_{p}^{s}\left(\mathbb{R}^{d}\right)$ with $s>1+d / p$, see [16]. Later, Vishik [21, 22] proved the global existence and uniqueness for 2D Euler equations in the borderline Besov spaces $B_{p, 1}^{1+2 / p}$ with $1<p<\infty$. Local existence and uniqueness was then extended to critical Besov space $B_{\infty, 1}^{1}\left(\mathbb{R}^{d}\right), d \geq 2$ by Pak and Park [17], see also [8] for a systematic treatment in Besov spaces. Recently, in [3, 4], Bourgain and Li proved a strongly ill-posedness result for the 2D or 3D Euler equations associated with initial data in Besov space $B_{p, q}^{d / p+1}$ for $1 \leq p<\infty, 1<q \leq \infty$ or Sobolev space $W^{d / p+1, p}$ with $1 \leq p<\infty$. For Euler equations, Himonas and Misiołek [13] proved the non-uniform dependence of the solution maps in $H^{s}\left(\mathbb{R}^{d}\right)$ with $s>0$. So one can only expect continuous dependence. Indeed, the continuous dependence in the Besov space, in particular $B_{\infty, 1}^{1}\left(\mathbb{R}^{d}\right)$, was shown recently in [12] using Bona-Smith $\operatorname{method}([5])$.

The existence and uniqueness of the Euler equations in general Triebel-Lizorkin spaces was studied by Chae, first in the subcritical space [6], and then in the critical space $([7]) F_{1, q}^{d+1}\left(\mathbb{R}^{d}\right)$ for $1 \leq q \leq \infty$. It is worth noting that a gap in [6] (on the trajectory mapping) was filled by [9]. It seems to us that the proof of a crucial proposition (Proposition 2.1) and commutator estimate (3.9) in [7] also have gaps. The main problem is that the critical space is now $L^{1}$-based for which usual technique may fail. For example, the (vector-valued) Hardy-Littlewood maximal

2010 Mathematics Subject Classification. 35Q31, 76B03.

Key words and phrases. Euler equations, commutator estimates, continuous dependence.

* Corresponding author. 
operator used in $[6,9]$ is not $L^{1}$ bounded. On the other hand, in [19], some counterexamples of commutator estimates in the Besov and the Triebel-Lizorkin spaces were constructed. In particular,

$$
\left\|2^{j s}\left[u, \Delta_{j}\right] \cdot v\right\|_{L_{x}^{p} q_{j}^{q}(\mathbb{Z})} \leq C\|u\|_{F_{p, q}^{s}}\|v\|_{F_{p, q}^{s}}, \quad \operatorname{div} u=0,
$$

fails for $1 \leq p<\infty, 1 \leq q \leq \infty, s<1+d / p$. However, we shall prove (1.2) holds with $p=1,1 \leq q \leq \infty, s=d+1$, see Proposition 2.11.

The purpose of this paper is twofold. First, we fill the gap in [7] and prove relevant estimates in the endpoint Triebel-Lizorkin spaces $F_{p, q}^{s}$ with $p=1$. To do this, we used some new techniques regarding maximal function estimates from [20]. Second, we show the continuous dependence in the (critical) Triebel-Lizorkin space using Bona-Smith method as in [12]. This together with the previous results $[6,7,9]$ implies the well-posedness of the Euler equations in these spaces in the sense of Hadamard. The main result of this paper is

Theorem 1.1. Assume that $d \geq 2,(s, p, q)$ satisfies

$$
s>\frac{d}{p}+1,(p, q) \in(1, \infty) \times(1, \infty) \quad \text { or } \quad s \geq d+1, p=1, q \in[1, \infty) .
$$

Then for arbitrary $R>0, u_{0} \in D(R):=\left\{\phi \in F_{p, q}^{s}:\|\phi\|_{F_{p, q}^{s}} \leq R, \operatorname{div} \phi=0\right\}$, there exist some $T=T(R, s, p, q, d)>0$ and a unique solution $u:=S_{T}\left(u_{0}\right) \in$ $C\left([0, T] ; F_{p, q}^{s}\right)$ to the Euler equations. Moreover, it satisfies

(1) (Boundedness): there exists some $C=C(s, p, q, d)$, such that

$$
\left\|S_{T}\left(u_{0}\right)\right\|_{L_{T}^{\infty} F_{p, q}^{s}} \leq C\left\|u_{0}\right\|_{F_{p, q}^{s}} .
$$

(2) (Continuous dependence): the solution map $u_{0} \rightarrow S_{T}\left(u_{0}\right)$ is continuous from $D(R)$ to $C\left([0, T] ; F_{p, q}^{s}\right)$. Precisely, for any $\epsilon>0$, there exists $\eta=$ $\eta\left(u_{0}, R, s, p, q, d\right)$ such that for any $\psi \in D(R)$ with $\left\|\psi-u_{0}\right\|_{F_{p, q}^{s}}<\eta$, then

$$
\left\|S_{T}\left(u_{0}\right)-S_{T}(\psi)\right\|_{L_{T}^{\infty} F_{p, q}^{s}}<\epsilon .
$$

Remark 1. Suppose $u_{0} \in F_{p, \infty}^{s}\left(\mathbb{R}^{d}\right)$ with $1<p<\infty, s>1+d / p$, one can also construct a unique local in time solution belonging to $L_{T}^{\infty} F_{p, \infty}^{s}$ for some $T=T\left(\left\|u_{0}\right\|_{F_{p, \infty}^{s}}\right)$. Local existence and uniqueness, and part (1) was obtained in $[6,7,9]$, except the case $s>d+1, p=1$ and $1 \leq q<\infty$, which seems to be new. For the convenience of reader and to make the paper more self-contained, we also provide a sketched proof in the appendix. The part (2) seems not proved before.

Remark 2. We remark that the above theorem also holds for the ideal MHD equations studied in [9]. The proof for MHD has slight difference. So our results extend the result of [9] to the critical space.

Our proof of Theorem 1.1 is conceptually similar to the one in [12], but the problem is technically harder. The main difficulty lies in establishing a Moser type inequality and a commutator estimate in the case of $p=1$. See Proposition 2.9 and Proposition 2.11 in Section 2.

Next we clarify some notations being used throughout this paper. $\mathcal{S}$ and $\mathcal{S}^{\prime}$ denote the set of Schwartz functions and tempered distributions over $\mathbb{R}^{d}$ respectively. $\mathscr{F} f=\hat{f}$ stands for the Fourier transform of $f$, and $\mathcal{F}^{-1} f=\check{f}$, the inverse Fourier transform of $f$. The symbol $C$ denotes a generic constant, which may be different from line to line. The function spaces are all defined over $\mathbb{R}^{d}$. For simplicity, the 
domain will often be omitted, e.g. we use $L^{p}$ instead of $L^{p}\left(\mathbb{R}^{d}\right)$ in many places, if not otherwise indicated. $B(x, r)$ means a ball centred at $x$ with radius $r$ and $B(r):=B(0, r)$.

Let us introduce the functional setting of this paper. Suppose $\varphi \in C^{\infty}\left(\mathbb{R}^{d}\right)$ satisfies $0 \leq \varphi \leq 1, \varphi=1$ on $B(1 / 2)$ and $\varphi=0$ outside $B(1)$. Set $\psi(\xi)=$ $\varphi(\xi / 2)-\varphi(\xi)$, we denote $\psi_{j}(\xi)=\psi\left(\xi / 2^{j}\right)$ and $\varphi_{j}(\xi)=\varphi\left(\xi / 2^{j}\right)$. The frequency localization operator is defined by

$$
\Delta_{j}:=\left(\mathscr{F}^{-1} \psi_{j}\right) *, \quad S_{j}=P_{\leq j}:=\left(\mathscr{F}^{-1} \varphi_{j}\right) *,
$$

here $*$ is the convolution operator in $\mathbb{R}^{d}$. It is easy to see $\Delta_{j}=S_{j+1}-S_{j}$. For $1 \leq p<\infty, 1 \leq q \leq \infty$, the inhomogeneous Triebel-Lizorkin spaces $F_{p, q}^{s}=F_{p, q}^{s}\left(\mathbb{R}^{d}\right)$ is defined by

$$
F_{p, q}^{s}:=\left\{f \in \mathcal{S}^{\prime}\left(\mathbb{R}^{d}\right), \quad\|f\|_{F_{p, q}^{s}}<\infty\right\},
$$

where

$$
\|f\|_{F_{p, q}^{s}}:=\left\|\left(\left|P_{\leq 0} f\right|^{q}+\sum_{j \geq 0} 2^{j s q}\left|\Delta_{j} f\right|^{q}\right)^{\frac{1}{q}}\right\|_{L_{x}^{p}},
$$

with the usual modification when $q=\infty$. Let $\mathcal{S}^{\prime} \backslash \mathcal{P}$ denote the tempered distribution modulo the polynomials, then

$$
\dot{F}_{p, q}^{s}:=\left\{f \in \mathcal{S}^{\prime} \backslash \mathcal{P}, \quad\|f\|_{\dot{F}_{p, q}^{s}}<\infty\right\},
$$

where

$$
\|f\|_{\dot{F}_{p, q}^{s}}:=\left\|\left(\sum_{j \in \mathbb{Z}} 2^{j s q}\left|\Delta_{j} f\right|^{q}\right)^{\frac{1}{q}}\right\|_{L_{x}^{p}} .
$$

We remark that for any $s>0$,

$$
\|f\|_{F_{p, q}^{s}} \sim\|f\|_{L^{p}}+\|f\|_{F_{p, q}^{s},}, \quad 1 \leq p<\infty, \quad 1 \leq q \leq \infty .
$$

See e.g. [20, 23]. Analogously, for $1 \leq p, q \leq \infty$, we have

$$
\|f\|_{B_{p, q}^{s}}:=\left(\left\|P_{\leq 0} f\right\|_{L_{x}^{p}}^{q}+\sum_{j \geq 0} 2^{j s q}\left\|\Delta_{j} f\right\|_{L_{x}^{p}}^{q}\right)^{\frac{1}{q}},
$$

and

$$
\|f\|_{\dot{B}_{p, q}^{s}}:=\left(\sum_{j \in \mathbb{Z}} 2^{j s q}\left\|\Delta_{j} f\right\|_{L_{x}^{p}}^{q}\right)^{\frac{1}{q}} .
$$

We refer reader to $[1,20,23]$ for more introductions on these function spaces.

The remaining part of this paper is structured as follows. In Section 2, we list some well known results and prove the key estimates for the proof. Section 3 is devoted to proving Theorem 1.1. Finally, we include an appendix, where local Cauchy theory for Euler equations in Triebel-Lizorkin spaces is given. 


\section{Auxiliary Results}

In this section, we recall some well-known facts and present several results which will be used in the sequel.

Lemma 2.1. Let $1 \leq p_{0}<p_{1}<\infty, 1 \leq q_{0} \leq \infty$ and $s_{0}-d / p_{0}=s_{1}-d / p_{1}$, then the following continuous embeddings hold:

$$
\dot{F}_{p_{0}, q_{0}}^{s_{0}} \hookrightarrow \dot{B}_{p_{1}, p_{0}}^{s_{1}}, \quad F_{p_{0}, q_{0}}^{s_{0}} \hookrightarrow B_{p_{1}, p_{0}}^{s_{1}} .
$$

For the proof, one can refer to [14]. As a simple consequence, we have

$$
\|f\|_{L^{\infty}} \leq\|f\|_{B_{\infty, 1}^{0}} \leq C\|f\|_{F_{1, \infty}^{d}}, \quad\|\nabla f\|_{L^{\infty}} \leq\|f\|_{B_{\infty, 1}^{1}} \leq C\|f\|_{F_{1, \infty}^{d+1}} .
$$

The following is a lifting property of the homogeneous Triebel-Lizorkin spaces, whose proof can be found in $[11,20]$.

Lemma 2.2. For any $k \in \mathbb{N},(p, q) \in[1, \infty) \times[1, \infty]$ and $s \in \mathbb{R}$, we have

$$
c\left\|D^{k} f\right\|_{\dot{F}_{p, q}^{s}} \leq\|f\|_{\dot{F}_{p, q}^{s+k}} \leq C\left\|D^{k} f\right\|_{\dot{F}_{p, q}^{s}}
$$

holds for some constant $c, C$, here $D:=\sqrt{-\Delta}$.

Following the definition, we have for $s \in \mathbb{R}$,

$$
\|f\|_{F_{p, q}^{s}} \leq\left\|P_{\leq 0} f\right\|_{L^{p}}+\|f\|_{\dot{F}_{p, q}^{s}}, \quad 1 \leq p<\infty, \quad 1 \leq q \leq \infty .
$$

When treating Euler equations in $F_{p, q}^{s}$, we should take caution to deal with the low frequency estimate in $L^{p}$ (particularly when $p=1$ ) spaces for the pressure term, a kernel property needs to be exploited(see a different treatment in [17]), which reads

Lemma 2.3. Let $m(\xi)$ be the Fourier symbol of operator $P_{\leq 0}(-\Delta)^{-1} \partial_{l} \partial_{k}, 1 \leq k, l \leq$ $d$, Then there exists a constant $C$, such that

$$
\left\|\mathscr{F}^{-1}\left(m(\xi) \xi_{i}\right)\right\|_{L^{1}} \leq C, \quad \forall 1 \leq i \leq d .
$$

Proof. Since $\Delta_{j} P_{\leq 0}(-\Delta)^{-1} \partial_{l} \partial_{l}=0$ if $j \geq 1$, we have

$$
\begin{aligned}
\left\|\mathscr{F}^{-1}\left(m(\xi) \xi_{i}\right)\right\|_{L^{1}} & \leq \sum_{j \leq 0}\left\|\mathscr{F}^{-1}\left(m(\xi) \psi_{j}(\xi) \xi_{i}\right)\right\|_{L^{1}} \\
& \leq \sum_{j \leq 0} 2^{j}\left\|\mathscr{F}^{-1}\left(m\left(2^{j} \xi\right) \psi(\xi) \xi_{i}\right)\right\|_{L^{1}}
\end{aligned}
$$

While according to Bernstein multiplier theorem (see [23], p.7),

$$
\left\|\mathscr{F}^{-1}\left(m\left(2^{j} \xi\right) \psi(\xi) \xi_{i}\right)\right\|_{L^{1}} \leq C\left\|m\left(2^{j} \xi\right) \psi(\xi) \xi_{i}\right\|_{H^{L}}, \quad L=[d / 2]+1 .
$$

As $\operatorname{supp} \psi \subset\left\{\xi \in \mathbb{R}^{d}: 1 / 2 \leq|\xi| \leq 2\right\}$, by a direct calculation, one can assert that there exists some constant $C$ independent of $j$, verifying

$$
\left\|m\left(2^{j} \xi\right) \psi(\xi) \xi_{i}\right\|_{H^{L}} \leq C\left(\left\|m\left(2^{j} \xi\right) \psi(\xi) \xi_{i}\right\|_{L^{2}}+\sum_{|\alpha|=L}\left\|\partial_{\xi}^{\alpha}\left(m\left(2^{j} \xi\right) \psi(\xi) \xi_{i}\right)\right\|_{L^{2}}\right) \leq C .
$$

This combined with (2.3) implies the desired result.

We will also need the Hardy-Littlewood maximal function. For a locally integrable function $f$ in $\mathbb{R}^{d}$, the maximal function $M f(x)$ is defined by

$$
M f(x)=\sup _{r>0} \frac{1}{|B(x, r)|} \int_{B(x, r)}|f(y)| d y .
$$


In addition, suppose $\Omega \subset \mathbb{R}^{d}$ is a compact set, we denote

$$
\mathcal{S}^{\Omega}=\left\{f \in \mathcal{S}\left(\mathbb{R}^{d}\right), \operatorname{supp} \hat{f} \subset \Omega\right\}, \quad L_{p}^{\Omega}=\left\{f \in L^{p}\left(\mathbb{R}^{d}\right), \text { supp } \hat{f} \subset \Omega\right\} .
$$

Below we recall a lemma on the pointwise estimate in terms of the maximal function, for the proof, see $[20]$ p.16.

Lemma 2.4. Let $f \in \mathcal{S}^{B(1)}, 0<r<\infty$, then there exists some constant $C$, such that

$$
\sup _{y \in \mathbb{R}^{d}} \frac{|f(x-y)|}{1+|y|^{\frac{d}{r}}} \leq C\left[M\left(|f|^{r}\right)(x)\right]^{\frac{1}{r}}
$$

Remark 3. The above conclusion still holds for $f \in L_{p}^{B(1)}$, see [20], p.22. In addition, if $\operatorname{supp} \hat{f} \subset B(R)$, one can have

$$
\sup _{y \in \mathbb{R}^{d}} \frac{|f(x-y)|}{1+|R y|^{\frac{d}{r}}} \leq C\left[M\left(|f|^{r}\right)(x)\right]^{\frac{1}{r}} .
$$

here $C$ is independent of $R$. In fact, set $g_{R}(\cdot)=f\left(R^{-1} \cdot\right)$, then applying Lemma 2.4 to $g_{R}$ yields the desired result.

Next we recall the well-known pointwise maximal function estimate, see [18].

Lemma 2.5. Let $g(x)$ be a nonnegative radial decreasing integrable function, suppose $|\psi(x)| \leq g(x)$ almost everywhere and $f \in L_{l o c}^{1}\left(\mathbb{R}^{d}\right)$, then

$$
\left|\psi_{\epsilon} * f(x)\right| \leq C M(f)(x), \quad \forall \epsilon>0,
$$

where $\psi_{\epsilon}(x)=\epsilon^{-d} \psi\left(\epsilon^{-1} x\right), C=\|\psi\|_{L^{1}}$.

Proposition 2.6. Let $L>0, j, k \in \mathbb{Z}, j>k-L$ and $r \in(0, \infty) . \psi \in C^{\infty}\left(\mathbb{R}^{d}\right)$ satisfies

$$
|\psi(z)|\left(1+|z|^{\frac{d}{r}}\right) \leq g(z),
$$

where $g(z)$ is some nonnegative radial decreasing integrable function. Denote $\psi_{k}(x)=$ $2^{k d} \psi\left(2^{k} x\right)$, then for any $\theta \in(0,1]$, there exists a constant $C$ independent of $j, k$, such that the following inequality

$$
\left|\left(\psi_{k} * f\right)(x)\right| \leq C 2^{(j-k) \theta \frac{d}{r}} M\left(|f|^{1-\theta}\right)(x)\left[M\left(|f|^{r}\right)(x)\right]^{\frac{\theta}{r}}
$$

holds for all $f \in L_{p}^{B\left(c 2^{j}\right)}$ with $p \geq 1$ and some generic constant $c$.

Proof. Consider $f \in \mathcal{S}^{B\left(c 2^{j}\right)}$ first, we have

$$
\begin{aligned}
&\left|\left(\psi_{k} * f\right)(x)\right| \leq \int_{\mathbb{R}^{d}}|\psi(y)|\left|f\left(x-2^{-k} y\right)\right| d y \\
& \leq \\
& \leq\left[\int_{\mathbb{R}^{d}}|\psi(y)|\left|f\left(x-2^{-k} y\right)\right|^{1-\theta}\left(1+\left(2^{j-k}|y|\right)^{\theta d / r}\right) d y\right] \\
& \quad \times \sup _{y \in \mathbb{R}^{d}} \frac{\left|f\left(x-2^{-k} y\right)\right|^{\theta}}{1+\left(2^{j-k}|y|\right)^{\theta d / r}} .
\end{aligned}
$$

In view of $(3)$, one can see

$$
\sup _{y \in \mathbb{R}^{d}} \frac{\left|f\left(x-2^{-k} y\right)\right|^{\theta}}{1+\left(2^{j-k}|y|\right)^{\theta d / r}} \leq C\left[M\left(|f|^{r}\right)(x)\right]^{\frac{\theta}{r}} .
$$


Given that $j-k>-L$, then

$$
\begin{aligned}
& \int_{\mathbb{R}^{d}}|\psi(y)|\left|f\left(x-2^{-k} y\right)\right|^{1-\theta}\left(1+\left(2^{j-k}|y|\right)^{\theta d / r}\right) d y \\
& \leq C 2^{(j-k) \frac{\theta d}{r}} \int_{\mathbb{R}^{d}}|\psi(y)|\left|f\left(x-2^{-k} y\right)\right|^{1-\theta}\left(1+|y|^{\theta d / r}\right) d y \\
& \leq C 2^{(j-k) \frac{\theta d}{r}} M\left(|f|^{1-\theta}\right)(x) .
\end{aligned}
$$

where we used hypothesis (2.6) and Lemma 2.5 in the last inequality. Hence, the proof is completed for $f \in \mathcal{S}^{B\left(c 2^{j}\right)}$.

For general $f \in L_{p}^{B\left(c 2^{j}\right)}$, one can choose $\varphi \in \mathcal{S}$, such that $\varphi(0)=1$ and $\operatorname{supp} \hat{\varphi} \subset$ $B(1)$. Denote $f_{\delta}:=\varphi(\delta x) f(x)$, applying previous result to $f_{\delta}$ and letting $\delta \rightarrow 0$, one can find (2.7) follows, see also [20] (p.22) for more explanations.

Remark 4. One can easily see from the above proof

$$
\left|\left(\psi_{k} * f\right)(x)\right| \leq C_{L}\left[M\left(|f|^{r}\right)(x)\right]^{\frac{1}{r}},
$$

provided $j \leq k+L$. Then for $1 \leq p<\infty, 1 \leq q \leq \infty$, it follows

$$
\left\|P_{\leq m} f\right\|_{F_{p, q}^{s+l}} \leq C 2^{m l}\|f\|_{F_{p, q}^{s}}, \quad \forall m, l \geq 0 .
$$

The following vector-valued maximal function estimate will also be frequently used, see $[10,18]$ for a proof.

Proposition 2.7. Let $(p, q) \in(1, \infty) \times(1, \infty]$ or $p=q=\infty$ be given. Suppose $\left\{f_{j}\right\}_{j \in \mathbb{Z}}$ is a sequence of functions in $L^{p}\left(\mathbb{R}^{d}\right)$ satisfying $\left\|f_{j}\right\|_{l_{j}^{q}(\mathbb{Z})} \in L^{p}\left(\mathbb{R}^{d}\right)$, then

$$
\left\|M\left(f_{j}\right)(x)\right\|_{L_{x}^{p} l_{j}^{q}} \leq C\left\|f_{j}(x)\right\|_{L_{x}^{p} l_{j}^{q}}
$$

for some constant $C=C(p, q)$.

Next we establish the Moser type inequality for the Triebel-Lizorkin spaces. First we recall

Proposition $2.8([6])$. Let $(p, q) \in(1, \infty) \times(1, \infty]$ or $p=q=\infty, s>0$. There exists some positive constant $C$ with the following property:

$$
\|f g\|_{\dot{F}_{p, q}^{s}} \leq C\left(\|f\|_{L^{\infty}}\|g\|_{\dot{F}_{p, q}^{s}}+\|g\|_{L^{\infty}}\|f\|_{\dot{F}_{p, q}^{s}}\right) \text {. }
$$

Proposition 2.1 in [7] claimed that the above proposition also holds for $s>0, p=$ $1,1 \leq q \leq \infty$. However, the proof of Proposition 2.1 in [7] seems to have gaps. In the following proposition, we re-prove the endpoint case $p=1$, which exactly complements the nonendpoint conuterpart.

Proposition 2.9 (Endpoint case). Let $q \in[1, \infty]$ be given, then there exists some constant $C$ such that

$$
\|f g\|_{\dot{F}_{1, q}^{s}} \leq C\left(\|f\|_{L^{\infty}}\|g\|_{\dot{F}_{1, q}^{s}}+\|f\|_{\dot{F}_{1, q}^{s}}\|g\|_{L^{\infty}}\right), \quad s>0
$$

holds for scalar functions $f$ and $g$. Additionally, suppose that $v$ is a scalar function and $u$ is a vector-valued function with $\operatorname{div} u=0$, then

$$
\|u \cdot \nabla v\|_{\dot{F}_{1, q}^{s}} \leq C\left(\|u\|_{L^{\infty}}\|\nabla v\|_{\dot{F}_{1, q}^{s}}+\|\nabla v\|_{L^{\infty}}\|u\|_{\dot{F}_{1, q}^{s}}\right), \quad s>-1 .
$$

and

$$
\|u \cdot \nabla v\|_{\dot{F}_{1, q}^{s}} \leq C\left(\|u\|_{L^{\infty}}\|\nabla v\|_{\dot{F}_{1, q}^{s}}+\|v\|_{L^{\infty}}\|\nabla u\|_{\dot{F}_{1, q}^{s}}\right), \quad s>-1 .
$$


Proof. We use the following Bony decomposition ([2])

$$
f g=T_{f} g+T_{g} f+R(f, g),
$$

where

$$
T_{f} g=\sum S_{j-3} f \Delta_{j} g=\sum_{j \in \mathbb{Z}} \sum_{l \leq j-4} \Delta_{l} f \Delta_{j} g, \quad R(f, g)=\sum_{|j-k| \leq 3} \Delta_{j} f \Delta_{k} g .
$$

Due to frequency interaction, one can figure out that $\Delta_{m}\left(S_{j-3} f \Delta_{j} g\right)=0$ if $|j-m| \geq$ 3 , hence for any $0<r<\infty$,

$$
\begin{aligned}
\left|2^{m s} \Delta_{m} T_{f} g\right| & =\left|2^{m s} \sum_{|j-m| \leq 2} \Delta_{m}\left(S_{j-3} f \Delta_{j} g\right)\right| \\
& \leq C \sum_{|a| \leq 2} 2^{m s}\left[M\left(\left|S_{m+a-3} f \Delta_{m+a} g\right|^{r}\right)(x)\right]^{\frac{1}{r}},
\end{aligned}
$$

where we used Proposition 2.6 with $\theta=1$. As such, choosing $0<r<1$ and applying Proposition 2.7, we have

$$
\begin{aligned}
\left\|T_{f} g\right\|_{\dot{F}_{1, q}^{s}} & \leq C \sum_{|a| \leq 2}\left\|M\left(\left|\left(S_{m+a-3} f\right)\left(2^{m s} \Delta_{m+a} g\right)\right|^{r}\right)(x)\right\|_{L_{x}^{1 / r} l_{m}^{q / r}}^{\frac{1}{r}} \\
& \leq C \sum_{|a| \leq 2}\left\|2^{m s}\left|S_{m+a-3} f \Delta_{m+a} g\right|(x)\right\|_{L_{x}^{1} l_{m}^{q}} \\
& \leq C\|f\|_{L^{\infty}}\|g\|_{\dot{F}_{1, q}^{s}} .
\end{aligned}
$$

Similarly,

$$
\left\|T_{g} f\right\|_{\dot{F}_{1, q}^{s}} \leq C\|g\|_{L^{\infty}}\|f\|_{\dot{F}_{1, q}^{s}} .
$$

Now we estimate $R(f, g)=\sum_{|b| \leq 3} \sum_{j \in \mathbb{Z}} \Delta_{j} f \Delta_{j+b} g$. For arbitrary fixed $r \in(0,1)$, as $s>0$, we can specify $\theta \in(0,1)$ such that $s>d \theta / r$. Using the property of frequency support, one can assert that there exists a constant $L$, such that

$$
\begin{aligned}
\|R(f, g)\|_{\dot{F}_{1, q}^{s}} & \leq \sum_{|b| \leq 3}\left\|\sum_{j>m-L} 2^{m s} \Delta_{m}\left(\Delta_{j} f \Delta_{j+b} g\right)\right\|_{L_{x}^{1} l_{m}^{q}} \\
& \left.\leq C \sum_{|b| \leq 3} \| \sum_{j>m-L} 2^{(m-j)(s-\theta d / r)} M\left(\left|2^{j s} \Delta_{j} f \Delta_{j+b} g\right|^{1-\theta}\right)(x) \mid\right) \\
& \times\left[M\left(\left|2^{j s} \Delta_{j} f \Delta_{j+b} g\right|^{r}\right)(x)\right]^{\frac{\theta}{r}} \|_{L_{x}^{1} l_{m}^{q}} \\
& \left.\leq C \sum_{|b| \leq 3} \| M\left(\left|2^{j s} \Delta_{j} f \Delta_{j+b} g\right|^{1-\theta}\right)(x) \mid\right)\left[M\left(\left|2^{j s} \Delta_{j} f \Delta_{j+b} g\right|^{r}\right)(x)\right]^{\frac{\theta}{r}} \|_{L_{x}^{1} l_{j}^{q}} \\
& \left.\leq C \sum_{|b| \leq 3} \| M\left(\left|2^{j s} \Delta_{j} f \Delta_{j+b} g\right|^{1-\theta}\right)(x) \mid\right) \|_{L_{x}^{\frac{1}{1-\theta}} l_{j}^{\frac{q}{1-\theta}}} \\
& \left.\times C \sum_{|b| \leq 3} \|\left|2^{j s} \Delta_{j} f \Delta_{j+b} g\right|^{1-\theta}\right)(x)\left|\left\|\left.\right|_{L_{x}^{\frac{1}{1-\theta}} l_{j}^{\frac{q}{1-\theta}}}\right\| M\left(\left|2^{j s} \Delta_{j} f \Delta_{j+b} g\right|^{r}\right)(x) \|_{L_{x}^{\frac{1}{r}} l_{j}^{\frac{q}{r}}}^{\frac{\theta}{r}}\right. \\
& \left.\leq C\|f\|_{L^{\infty}}\|g\|_{F_{1, q}^{s}} \cdot(2.13)\right]_{L_{x}^{\frac{1}{\theta}} l_{j}^{\frac{q}{\theta}}}
\end{aligned}
$$


where we utilized Proposition 2.6, Young's inequality and Proposition 2.7 from the second to the last inequality. This yields (2.10).

As to the proof of (2.11), we first note that $u \cdot \nabla v=u^{l} \partial_{l} v$, here summation over repeated indices is adopted. Similarly,

$$
u \cdot \nabla v=T_{u^{l}} \partial_{l} v+T_{\partial_{l}} u^{l}+R\left(u^{l}, \partial_{l} v\right)
$$

In view of the argument above, one can easily see

$$
\left\|T_{u^{l}} \partial_{l} v\right\|_{\dot{F}_{1, q}^{s}}+\left\|T_{\partial_{l} v} u^{l}\right\|_{\dot{F}_{1, q}^{s}} \leq C\left(\|u\|_{L^{\infty}}\|\nabla v\|_{\dot{F}_{1, q}^{s}}+\|\nabla v\|_{L^{\infty}}\|u\|_{\dot{F}_{1, q}^{s}}\right) .
$$

Thanks to the divergence free condition on $u$, we know $R\left(u^{l}, \partial_{l} v\right)=\partial_{l} R\left(u^{l}, v\right)$, by Lemma 2.2

$$
\left\|R\left(u^{l}, \partial_{l} v\right)\right\|_{\dot{F}_{1, q}^{s}} \leq C \sum_{1 \leq l \leq d}\left\|R\left(u^{l}, v\right)\right\|_{\dot{F}_{1, q}^{s+1}}
$$

Then the argument of $R(f, g)$ above implies that

$$
\left\|R\left(u^{l}, v\right)\right\|_{\dot{F}_{1, q}^{s+1}} \leq C\left\|u^{l}\right\|_{L^{\infty}}\|\nabla v\|_{\dot{F}_{1, q}^{s}}
$$

holds for all $s>-1$. Hence, (2.11) is proved. Finally, owing to Lemma 2.2,

$$
\|u \cdot \nabla v\|_{\dot{F}_{1, q}^{s}} \leq C \sum_{1 \leq l \leq d}\left\|u^{l} v\right\|_{\dot{F}_{1, q}^{s+1}}
$$

Thus (2.12) is a simple consequence of (2.10).

We shall conclude this section by presenting the commutator estimates, which turns out to be an important tool in [16]. In order to estimate the $F_{p, q}^{s}$ norm of the solution to the Euler equations, a commutator involve frequency localization operator occurs naturally, Let us first recall that

$$
\left[f, \Delta_{j}\right] g:=f \Delta_{j} g-\Delta_{j}(f g) .
$$

Proposition $2.10([9])$. Let $(p, q) \in(1, \infty) \times(1, \infty]$. Suppose $f$ is a divergence free vector field, then there exists a constant $C$, such that for $s>0$,

$$
\left\|2^{j s}\left[f, \Delta_{j}\right] \cdot \nabla g\right\|_{L_{x}^{p} l_{j}^{q}} \leq C\left(\|\nabla f\|_{L^{\infty}}\|g\|_{\dot{F}_{p, q}^{s}}+\|\nabla g\|_{L^{\infty}}\|f\|_{\dot{F}_{p, q}^{s}}\right) .
$$

or for $s>-1$,

$$
\left\|2^{j s}\left[f, \Delta_{j}\right] \cdot \nabla g\right\|_{L_{x}^{p} l_{j}^{q}} \leq C\left(\|\nabla f\|_{L^{\infty}\|g\|_{\dot{F}_{p, q}^{s}}}+\|g\|_{L^{\infty}}\|\nabla f\|_{\dot{F}_{p, q}^{s}}\right) .
$$

Proposition 2.11 (Endpoint commutator estimate). Let $d \geq 1$ denote the space dimension, $q \in[1, \infty]$ be given. There exists a constant $C$, such that

$$
\left\|2^{j s}\left[f, \Delta_{j}\right] \cdot \nabla g\right\|_{L_{x}^{1} l_{j}^{q}} \leq C\left(\|\nabla f\|_{L^{\infty}}\|g\|_{\dot{F}_{1, q}^{s}}+\|\nabla g\|_{L^{\infty}}\|f\|_{\dot{F}_{1, q}^{s}}\right), \quad s>0,
$$

and

$$
\left\|2^{j s}\left[f, \Delta_{j}\right] \cdot \nabla g\right\|_{L_{x}^{1} l_{j}^{q}} \leq C\left(\|\nabla f\|_{L^{\infty}}\|g\|_{\dot{F}_{1, q}^{s}}+\|g\|_{L^{\infty}}\|\nabla f\|_{\dot{F}_{1, q}^{s}}\right), \quad s>-1
$$

hold for all scalar function $g$ and vector-valued function $f$ with $\operatorname{div} f=0$.

Proof. We first show (2.14). Let $f=\left(f^{l}\right)_{1 \leq l \leq d}$, according to Bony decomposition, one can see

$$
\begin{aligned}
{\left[f, \Delta_{j}\right] \cdot \nabla g } & =f^{l} \Delta_{j} \partial_{l} g-\Delta_{j}\left(f^{l} \partial_{l} g\right) \\
& =T_{\Delta_{j} \partial_{l} g} f^{l}+R\left(f^{l}, \Delta_{j} \partial_{l} g\right)+\left[T_{f^{l}}, \Delta_{j}\right] \partial_{l} g-\Delta_{j} T_{\partial_{l} g} f^{l}-\Delta_{j} R\left(f^{l}, \partial_{l} g\right) .
\end{aligned}
$$


It suffices to bound the above five terms in turn. Note that $S_{m-3} \Delta_{j}=0$ if $m \leq j+2$, thus

$$
\begin{aligned}
\left\|2^{j s} T_{\Delta_{j} \partial_{l} g} f^{l}\right\|_{L_{x}^{1} l_{j}^{q}} & =\left\|\sum_{m>j+2} 2^{(j-m) s}\left(S_{m-3} \Delta_{j} \partial_{l} g\right)\left(2^{m s} \Delta_{m} f^{l}\right)\right\|_{L_{x}^{1} l_{j}^{q}} \\
& \leq C\|\nabla g\|_{L^{\infty}} \sum_{1 \leq l \leq d}\left\|\sum_{m>j+2} 2^{(j-m) s} 2^{m s} \mid \Delta_{m} f^{l}\right\|_{L_{x}^{1} l_{j}^{q}} \\
& \leq C\|\nabla g\|_{L^{\infty}}\|f\|_{\dot{F}_{1, q}^{s}} .
\end{aligned}
$$

where we used Young's inequality in the last step as $s>0$. On the estimate of $R\left(f^{l}, \Delta_{j} \partial_{l} g\right)$, one can see

$$
\left\|2^{j s} R\left(f^{l}, \Delta_{j} \partial_{l} g\right)\right\|_{L_{x}^{1} l_{j}^{q}} \leq \sum_{|a| \leq 3} \sum_{|j-m| \leq 1}\left\|2^{j s} \partial_{l}\left[\left(\Delta_{m+a} f^{l}\right) \Delta_{m}\left(\Delta_{j} g\right)\right]\right\|_{L_{x}^{1} l_{j}^{q}} .
$$

Let $\phi \in C_{0}^{\infty}\left(\mathbb{R}^{d}\right), \phi=1$ on $B\left(2^{5}\right)$. Due to the fact

$$
\operatorname{supp} \mathscr{F}\left(\Delta_{m+a} f^{l} \Delta_{m} \Delta_{j} g\right) \subset B\left(2^{m+5}\right) \text {. }
$$

We can assert

$$
\partial_{l}\left(\Delta_{m+a} f^{l} \Delta_{m} \Delta_{j} g\right)=\check{\phi}_{m} * \partial_{l}\left(\Delta_{m+a} f^{l} \Delta_{m} \Delta_{j} g\right), \quad \check{\phi}_{m}(\cdot):=2^{m d} \check{\phi}\left(2^{m} \cdot\right) .
$$

Now applying Proposition 2.6 with $\theta=1$ and Proposition 2.7, we get

$$
\begin{aligned}
& \left\|2^{j s} R\left(f^{l}, \Delta_{j} \partial_{l} g\right)\right\|_{L_{x}^{1} l_{j}^{q}} \\
& \leq C \sum_{1 \leq l \leq d|a| \leq 3} \sum_{|b| \leq 1}\left\|\left[M\left(\left|2^{j} \Delta_{j+a+b} f^{l} \| \Delta_{j+b}\left(2^{j s} \Delta_{j} g\right)\right|\right)^{r}(x)\right]^{\frac{1}{r}}\right\|_{L_{x}^{1} l_{j}^{q}} \\
& \leq C\|\nabla f\|_{L^{\infty}}\|g\|_{\dot{F}_{1, q}^{s}} .
\end{aligned}
$$

where we used the following simple fact

$$
2^{j}\left\|\Delta_{j+b} f\right\|_{L^{\infty}} \leq C_{b}\|\nabla f\|_{L^{\infty}} .
$$

Concerning the third term $\left[T_{f^{l}}, \Delta_{j}\right] \partial_{l} g$, we first note that

$$
\left[T_{f^{l}}, \Delta_{j}\right] \partial_{l} g=\sum_{m \in \mathbb{Z}}\left[S_{m-3} f^{l}, \Delta_{j}\right] \Delta_{m} \partial_{l} g=\sum_{|m-j| \leq 2}\left[S_{m-3} f^{l}, \Delta_{j}\right] \Delta_{m} \partial_{l} g .
$$

Furthermore,

$$
\begin{aligned}
& \sum_{|m-j| \leq 2}\left|\left[S_{m-3} f^{l}, \Delta_{j}\right] \Delta_{m} \partial_{l} g\right| \\
= & \sum_{|a| \leq 2}\left|\int_{\mathbb{R}^{d}} 2^{j d} \check{\psi}\left(2^{j}(x-y)\right)\left(S_{j+a-3} f^{l}(x)-S_{j+a-3} f^{l}(y)\right) \Delta_{j+a} \partial_{l} g(y) d y\right| \\
\leq & \sum_{|a| \leq 2} \int_{\mathbb{R}^{d}} 2^{j(d+1)}\left|\left(\partial_{l} \check{\psi}\right)\left(2^{j}(x-y)\right)\left(S_{j+a-3} f^{l}(x)-S_{j+a-3} f^{l}(y)\right) \Delta_{j+a} g(y)\right| d y \\
\leq & C \sum_{|a| \leq 2}\left\|\nabla S_{j+a-3} f^{l}\right\|_{L^{\infty}} \int_{\mathbb{R}^{d}} 2^{j(d+1)}|x-y|\left|\left(\partial_{l} \check{\psi}\right)\left(2^{j}(x-y)\right)\right|\left|\Delta_{j+a} g(y)\right| d y \\
\leq & C \sum_{|a| \leq 2}\|\nabla f\|_{L^{\infty}}\left[M\left(\left|\Delta_{j+a} g\right|^{r}\right)(x)\right]^{\frac{1}{r}} .
\end{aligned}
$$


here we used the $\operatorname{div} f=0$, mean value theorem and Proposition 2.6 with $\theta=1$ from the second to the fourth step. Therefore, it follows from Proposition 2.7 that

$$
\begin{aligned}
\left\|2^{j s}\left[T_{f^{l}}, \Delta_{j}\right] \partial_{l} g\right\|_{L_{x}^{1} l_{j}^{q}} & \leq C \sum_{|a| \leq 2}\|\nabla f\|_{L^{\infty}}\left\|\left[M\left(\left|2^{j s} \Delta_{j+a} g\right|^{r}\right)(x)\right]^{\frac{1}{r}}\right\|_{L_{x}^{1} l_{j}^{q}} \\
& \leq C\|\nabla f\|_{L^{\infty}}\|g\|_{\dot{F}_{1, q}^{s}}
\end{aligned}
$$

Regarding the term $\Delta_{j} T_{\partial_{l} g} f^{l}$, applying Proposition 2.6, we have

$$
\begin{aligned}
\left\|2^{j s} \Delta_{j} T_{\partial_{l} g} f^{l}\right\|_{L_{x}^{1} l_{j}^{q}} & =\left\|2^{j s} \sum_{|m-j| \leq 2} \Delta_{j}\left(\left(S_{m-3} \partial_{l} g\right) \Delta_{m} f^{l}\right)\right\|_{L_{x}^{1} l_{j}^{q}} \\
& \leq C \sum_{|a| \leq 2} \|\left[M\left(\left|\left(S_{j+a-3} \partial_{l} g\right)\left(2^{j s} \Delta_{j+a} f^{l}\right)\right|^{r}(x)\right]^{\frac{1}{r}} \|_{L_{x}^{1} l_{j}^{q}}\right. \\
& \leq C \sum_{|a| \leq 2} \sum_{1 \leq l \leq d}\|\nabla g\|_{L^{\infty}}\left\|2^{j s} \Delta_{j+a} f^{l}\right\|_{L_{x}^{1} l_{j}^{q}} \\
& \leq C\|\nabla g\|_{L^{\infty}}\|f\|_{\dot{F}_{1, q}^{s}} .
\end{aligned}
$$

Finally, we estimate the term $\Delta_{j} R\left(f^{l}, \partial_{l} g\right)$. Since $s>-1$, for arbitrary $r \in(0,1)$, one can select $\theta \in(0,1)$ small enough, such that $s+1>d \theta / r$. Due to frequency interaction, one can observe that there exists a constant $L$, such that

$$
\begin{aligned}
&\left|\Delta_{j} R\left(f^{l}, \partial_{l} g\right)\right|=\left|\sum_{|a| \leq 3} \sum_{m \geq j-L} \Delta_{j} \partial_{l}\left(\Delta_{m} f^{l} \Delta_{m+a} g\right)\right| \\
& \leq \sum_{1 \leq l \leq d} \sum_{|a| \leq 3} \sum_{m \geq j-L} C 2^{j} 2^{(m-j) \frac{d \theta}{r}} M\left(\left|\Delta_{m} f^{l} \Delta_{m+a} g\right|^{1-\theta}\right) \\
& \times\left[M\left(\left|\Delta_{m} f^{l} \Delta_{m+a} g\right|^{r}\right)(x)\right]^{\frac{\theta}{r}} .
\end{aligned}
$$

where Proposition 2.6 is used. Thanks to Young's inequality, one can get

$$
\begin{gathered}
\left\|2^{j s} \Delta_{j} R\left(f^{l}, \partial_{l} g\right)\right\|_{L_{x}^{1} l_{j}^{q}} \\
\leq C \sum_{1 \leq l \leq d} \sum_{|a| \leq 3} \| \sum_{m \geq j-L} 2^{(j-m)(s+1-d \theta / r)} M\left(\left|\left(2^{m} \Delta_{m} f^{l}\right)\left(2^{m s} \Delta_{m+a} g\right)\right|^{1-\theta}\right) \\
\times\left[M\left(\left|\left(2^{m} \Delta_{m} f^{l}\right)\left(2^{m s} \Delta_{m+a} g\right)\right|^{r}\right)(x)\right]^{\frac{\theta}{r}} \|_{L_{x}^{1} l_{j}^{q}} \\
\leq C \sum_{1 \leq l \leq d|a| \leq 3} \sum \| M\left(\left|\left(2^{m} \Delta_{m} f^{l}\right)\left(2^{m s} \Delta_{m+a} g\right)\right|^{1-\theta}\right) \\
\times\left[M\left(\left|\left(2^{m} \Delta_{m} f^{l}\right)\left(2^{m s} \Delta_{m+a} g\right)\right|^{r}\right)(x)\right]^{\frac{\theta}{r}} \|_{L_{x}^{1} l_{m}^{q}}
\end{gathered}
$$

Then one can argue analogously as (2.13) to obtain

$$
\left\|2^{j s} \Delta_{j} R\left(f^{l}, \partial_{l} g\right)\right\|_{L_{x}^{1} l_{j}^{q}} \leq C\|\nabla f\|_{L^{\infty}}\|g\|_{\dot{F}_{1, q}^{s}} .
$$

Gathering the estimates above, we find (2.14) follows. 
In order to show (2.15), it suffices to slightly modify the estimate of the terms $T_{\Delta_{j} \partial_{l} g} f^{l}$ and $\Delta_{j} T_{\partial_{l} g} f^{l}$. Note that $s>-1$, then

$$
\begin{aligned}
\left\|2^{j s} T_{\Delta_{j} \partial_{l} g} f^{l}\right\|_{L_{x}^{1} l_{j}^{q}} & =\left\|\sum_{m>j+2} 2^{(j-m) s}\left(S_{m-3} \Delta_{j} \partial_{l} g\right)\left(2^{m s} \Delta_{m} f^{l}\right)\right\|_{L_{x}^{1} l_{j}^{q}} \\
& \leq C\|\| \Delta_{j} \partial_{l} g\left\|_{L^{\infty}} \sum_{m>j+2} 2^{(j-m) s} \mid 2^{m s} \Delta_{m} f^{l}\right\| \|_{L_{x}^{1} l_{j}^{q}} \\
& \leq C\|g\|_{L^{\infty}} \sum_{1 \leq l \leq d}\left\|\sum_{m>j+2} 2^{(s+1)(j-m)} 2^{m(s+1)} \mid \Delta_{m} f^{l}\right\|_{L_{x}^{1} l_{j}^{q}} \\
& \leq C\|g\|_{L^{\infty}}\|f\|_{\dot{F}_{1, q}^{s+1}}
\end{aligned}
$$

where we used Young's inequality. Regarding to the term $\Delta_{j} T_{\partial_{l} g} f^{l}$, thanks to Proposition 2.6 and $\operatorname{div} u=0$, one can immediately have

$$
\begin{aligned}
\left\|2^{j s} \Delta_{j} T_{\partial_{l} g} f^{l}\right\|_{L_{x}^{1} l_{j}^{q}} & =\left\|2^{j s} \sum_{|m-j| \leq 2} \Delta_{j} \partial_{l}\left(\left(S_{m-3} g\right)\left(\Delta_{m} f^{l}\right)\right)\right\|_{L_{x}^{1} l_{j}^{q}} \\
& \leq C \sum_{|a| \leq 2} \sum_{1 \leq l \leq d}\left\|2^{(s+1) j}\left[M\left(\left|S_{j+a-3} g \Delta_{j+a} f^{l}\right|^{r}(x)\right)\right]^{\frac{1}{r}}\right\|_{L_{x}^{1} l_{j}^{q}} \\
& \leq C\|g\|_{L^{\infty}}\|f\|_{\dot{F}_{1, q}^{s+q}} .
\end{aligned}
$$

This completed the proof.

\section{Proof of the Main Result}

In this section, we follow the scheme of [12] to demonstrate that the solution map of Euler equations is continuous in Triebel-Lizorkin spaces.

Proof of Theorem 1.1. The proof is divided into four steps:

Step 1. It follows from local Cauchy theory that there exists some $T=T\left(\left\|u_{0}\right\|_{F_{p, q}^{s}}\right)$ and a unique solution $u=S_{T}\left(u_{0}\right) \in C\left([0, T] ; F_{p, q}^{s}\right)$ such that

$$
\|u\|_{L_{T}^{\infty} F_{p, q}^{s}} \leq C\left\|u_{0}\right\|_{F_{p, q}^{s}} .
$$

Moreover, if $u_{0} \in F_{p, q}^{s+\gamma}$ with some $\gamma>0$, then

$$
\|u\|_{L_{T}^{\infty} F_{p, q}^{s+\gamma}} \leq C\left\|u_{0}\right\|_{F_{p, q}^{s+\gamma}}
$$

One can refer to $[6,7]$ or Theorem A.1 in the Appendix for more details.

Step 2. For any $u_{0}, v_{0} \in D(R)=\left\{\psi \in F_{p, q}^{s}: \operatorname{div} \psi=0,\|\psi\|_{F_{p, q}^{s}} \leq R\right\}$, we have

$$
\left\|S_{T}\left(u_{0}\right)-S_{T}\left(v_{0}\right)\right\|_{L_{T}^{\infty} F_{p, q}^{s-1}} \leq C\left\|u_{0}-v_{0}\right\|_{F_{p, q}^{s-1}}
$$

In fact, let $u=S_{T}\left(u_{0}\right), v=S_{T}\left(v_{0}\right)$. Set $w=u-v$, then $w$ solves

$$
\left\{\begin{array}{l}
\partial_{t} w+w \cdot \nabla u+v \cdot \nabla w+\nabla(P(u)-P(v))=0, \\
\operatorname{div} w=0 \\
w(0, x)=w_{0}=u_{0}-v_{0} .
\end{array}\right.
$$

here $P(u):=(-\Delta)^{-1} \operatorname{div}(u \cdot \nabla u)$. Applying the frequency localization operator $\Delta_{j}$, one can find

$$
\partial_{t} \Delta_{j} w+v \cdot \nabla \Delta_{j} w+\Delta_{j}(w \cdot \nabla u)+\nabla \Delta_{j}(P(u)-P(v))=\left[v, \Delta_{j}\right] \cdot \nabla w .
$$


As in $[6,9]$, we introduce particle trajectory mapping $X(\alpha, t)$ defined by the solution of the ordinary differential equations

$$
\left\{\begin{array}{l}
\frac{\partial}{\partial t} X(\alpha, t)=v(X(\alpha, t), t) \\
X(\alpha, 0)=\alpha
\end{array}\right.
$$

This implies

$$
\begin{array}{r}
\Delta_{j} w(X(\alpha, t), t)=\Delta_{j} w_{0}(\alpha)+\int_{0}^{t}\left(\left[v, \Delta_{j}\right] \cdot \nabla w-\Delta_{j}(w \cdot \nabla u)\right)(X(\alpha, \tau), \tau) \\
-\nabla \Delta_{j}(P(u)-P(v))(X(\alpha, \tau), \tau) d \tau .
\end{array}
$$

Note that $\operatorname{div} v=0$, so $X(\alpha, t)$ is a measure preserving mapping. Multiplying $2^{j(s-1)}$ and taking $L_{\alpha}^{p} l_{j}^{q}$ norm on both sides of (3.4), we can see

$$
\begin{aligned}
\|w(t)\|_{\dot{F}_{p, q}^{s-1}} \leq\left\|w_{0}\right\|_{\dot{F}_{p, q}^{s-1}}+\int_{0}^{t} \| \nabla(P(u) & -P(v))\left\|_{\dot{F}_{p, q}^{s-1}}+\right\| w \cdot \nabla u \|_{\dot{F}_{p, q}^{s-1}} \\
& +\left\|2^{(s-1) j}\left[v, \Delta_{j}\right] \cdot \nabla w\right\|_{L_{x}^{p} l_{j}^{q}} d \tau .
\end{aligned}
$$

Similarly,

$$
w(X(\alpha, t), t)=w_{0}(\alpha)-\int_{0}^{t}(w \cdot \nabla u+\nabla(P(u)-P(v)))(X(\alpha, \tau), \tau) d \tau .
$$

which leads to

$$
\|w(t)\|_{L^{p}} \leq\left\|w_{0}\right\|_{L^{p}}+\int_{0}^{t}\|w \cdot \nabla u(\tau)\|_{L^{p}}+\|\nabla(P(u)-P(v))(\tau)\|_{L^{p}} d \tau .
$$

Combining the estimates (3.5) and (3.6), one can get

$$
\begin{array}{r}
\|w\|_{F_{p, q}^{s-1}} \leq\left\|w_{0}\right\|_{F_{p, q}^{s-1}}+\int_{0}^{t}\|w \cdot \nabla u\|_{F_{p, q}^{s-1}}+\|\nabla(P(u)-P(v))\|_{F_{p, q}^{s-1}} \\
+\left\|2^{j(s-1)}\left[v, \Delta_{j}\right] \cdot \nabla w\right\|_{L_{x}^{p} l_{j}^{q}} d \tau .
\end{array}
$$

Recall that for $a \in \mathbb{R}$, we have

$$
\|f\|_{F_{p, q}^{a}} \leq\left\|P_{\leq 0} f\right\|_{L^{p}}+\|f\|_{\dot{F}_{p, q}^{a}} .
$$

Noticing that

$$
\nabla(P(u)-P(v))=\nabla(-\Delta)^{-1} \operatorname{div}(w \cdot \nabla u+v \cdot \nabla w) .
$$

By Lemma 2.3, we can assert

$$
\left\|P_{\leq 0} \nabla(-\Delta)^{-1} \operatorname{div}(w \cdot \nabla u)\right\|_{L^{p}} \leq C\|w\|_{L^{\infty}}\|u\|_{L^{p}} .
$$

Owing to the boundedness of operator $\partial_{j} \partial_{k}(-\Delta)^{-1}$ in $\dot{F}_{p, q}^{s-1}($ see $[11,18,7])$ and Propositions 2.8 and 2.9, one can find

$$
\begin{aligned}
\left\|\nabla(-\Delta)^{-1} \operatorname{div}(w \cdot \nabla u)\right\|_{\dot{F}_{p, q}^{s-1}} & \leq C\|w \cdot \nabla u\|_{\dot{F}_{p, q}^{s-1}} \\
& \leq C\left(\|w\|_{L^{\infty}}\|u\|_{\dot{F}_{p, q}^{s}}+\|w\|_{\dot{F}_{p, q}^{s-1}}\|\nabla u\|_{L^{\infty}}\right) .
\end{aligned}
$$

Consequently,

$$
\left\|\nabla(-\Delta)^{-1} \operatorname{div}(w \cdot \nabla u)\right\|_{F_{p, q}^{s-1}} \leq C\|w\|_{F_{p, q}^{s-1}}\|u\|_{F_{p, q}^{s}}
$$


On the other hand, $\operatorname{div}(v \cdot \nabla w)=\operatorname{div}(w \cdot \nabla v)$, we finally obtain

$$
\|\nabla(P(u)-P(v))\|_{F_{p, q}^{s-1}} \leq C\|w\|_{F_{p, q}^{s-1}}\left(\|u\|_{F_{p, q}^{s}}+\|v\|_{F_{p, q}^{s}}\right) .
$$

In the same way, one can assert

$$
\|w \cdot \nabla u\|_{F_{p, q}^{s-1}} \leq C\|w\|_{F_{p, q}^{s-q}}\|u\|_{F_{p, q}^{s}} .
$$

Lastly, by virtue of Proposition 2.10 and Proposition 2.11,

$$
\begin{aligned}
\left\|2^{j(s-1)}\left[v, \Delta_{j}\right] \cdot \nabla w\right\|_{L_{x}^{p} l_{j}^{q}} & \leq C\left(\|\nabla v\|_{L^{\infty}}\|w\|_{\dot{F}_{p, q}^{s-1}}+\|w\|_{L^{\infty}}\|v\|_{\dot{F}_{p, q}^{s}}\right) \\
& \leq C\|w\|_{F_{p, q}^{s-1}}\|v\|_{F_{p, q}^{s} .}
\end{aligned}
$$

Summarizing the estimates above, we deduce

$$
\|w(t)\|_{F_{p, q}^{s-1}} \leq\left\|w_{0}\right\|_{F_{p, q}^{s-1}}+C \int_{0}^{t}\|w(\tau)\|_{F_{p, q}^{s-1}}\left(\|u(\tau)\|_{F_{p, q}^{s}}+\|v(\tau)\|_{F_{p, q}^{s}}\right) d \tau .
$$

Using (3.1) and Gronwall's inequality, one can have

$$
\|w\|_{L_{T}^{\infty} F_{p, q}^{s-1}} \leq C\left\|w_{0}\right\|_{F_{p, q}^{s-1}}
$$

which justifies (3.3).

Step 3. Let $u_{0} \in D(R)$, we claim

$$
\left\|S_{T}\left(u_{0}\right)-S_{T}\left(P_{\leq N} u_{0}\right)\right\|_{L_{T}^{\infty} F_{p, q}^{s}} \leq C\left\|u_{0}-P_{\leq N} u_{0}\right\|_{F_{p, q}^{s}} .
$$

For simplicity, let us denote $u=S_{T}\left(u_{0}\right), u^{N}=S_{T}\left(P_{\leq N} u_{0}\right)$ and $w^{N}=u-u^{N}$, according to the result in Step 1 and Remark 4, there exists some $T=T(R)$, such that

$$
\begin{aligned}
\|u\|_{L_{T}^{\infty} F_{p, q}^{s}}+\left\|u^{N}\right\|_{L_{T}^{\infty} F_{p, q}^{s}} & \leq C\left\|u_{0}\right\|_{F_{p, q}^{s}} \leq C, \\
\left\|u^{N}\right\|_{L_{T}^{\infty} F_{p, q}^{s+1}} & \leq C\left\|P_{\leq N} u_{0}\right\|_{F_{p, q}^{s+1}} \leq C 2^{N} .
\end{aligned}
$$

It is not hard to see $w^{N}$ is a solution of the following equation:

$$
\left\{\begin{array}{l}
\partial_{t} w^{N}+u \cdot \nabla w^{N}+w^{N} \cdot \nabla u^{N}+\nabla\left(P(u)-P\left(u^{N}\right)\right)=0, \\
\operatorname{div} w^{N}=0, \\
w^{N}(0, x)=w_{0}^{N}=u_{0}-P_{\leq N} u_{0} .
\end{array}\right.
$$

By means of argument similar to that in Step 2, one can deduce

$$
\begin{array}{r}
\left\|w^{N}(t)\right\|_{F_{p, q}^{s}} \leq\left\|w_{0}^{N}\right\|_{F_{p, q}^{s}}+\int_{0}^{t}\left\|w^{N} \cdot \nabla u^{N}\right\|_{F_{p, q}^{s}} \\
+\left\|\nabla\left(P(u)-P\left(u^{N}\right)\right)\right\|_{F_{p, q}^{s}} \\
+\left\|2^{j s}\left[u, \Delta_{j}\right] \cdot \nabla w^{N}\right\|_{L_{x}^{p} l_{j}^{q}} d \tau .
\end{array}
$$

Following (3.8), Lemma 2.3 and Propositions 2.8 and 2.9, we can get

$$
\begin{aligned}
\left\|w^{N} \cdot \nabla u^{N}\right\|_{F_{p, q}^{s}} & \leq C\left\|w^{N}\right\|_{L^{\infty}}\left\|u^{N}\right\|_{L^{p}}+C\left\|w^{N}\right\|_{L^{\infty}}\left\|\nabla u^{N}\right\|_{\dot{F}_{p, q}^{s}}+C\left\|w^{N}\right\|_{\dot{F}_{p, q}^{s}}\left\|\nabla u^{N}\right\|_{L^{\infty}} \\
& \leq C 2^{N}\left\|w^{N}\right\|_{F_{p, q}^{s-1}}+C\left\|w^{N}\right\|_{F_{p, q}^{s}}\left\|u^{N}\right\|_{F_{p, q}^{s}} \\
& \leq C\left\|w_{0}^{N}\right\|_{F_{p, q}^{s}}+C\left\|w^{N}\right\|_{F_{p, q}^{s}} .
\end{aligned}
$$

where we used the fact $2^{N}\left\|w_{0}^{N}\right\|_{F_{p, q}^{s-1}} \leq C\left\|w_{0}^{N}\right\|_{F_{p, q}^{s}}$ in the last inequality. Regarding the pressure,

$$
\nabla\left(P(u)-P\left(u^{N}\right)\right)=\nabla(-\Delta)^{-1} \operatorname{div}\left(w^{N} \cdot \nabla u^{N}+u \cdot \nabla w^{N}\right) .
$$


By Lemma 2.2 and the boundedness of Riesz operator in $\dot{F}_{p, q}^{s-1}$, one can have

$$
\begin{aligned}
& \left\|\nabla(-\Delta)^{-1} \operatorname{div}\left(w^{N} \cdot \nabla u^{N}\right)\right\|_{F_{p, q}^{s}} \\
& \leq C\left\|w^{N}\right\|_{L^{p}}\left\|u^{N}\right\|_{L^{\infty}}+C \sum_{1 \leq k \leq d}\left\|\left(\partial_{k} w^{N}\right) \cdot \nabla\left(u^{N}\right)^{k}\right\|_{\dot{F}_{p, q}^{s-1}} \\
& \leq C\left\|w^{N}\right\|_{F_{p, q}^{s}}\left\|u^{N}\right\|_{F_{p, q}^{s}} .
\end{aligned}
$$

where Propositions 2.8-2.9 is used in the last inequality, $\left(u^{N}\right)^{k}$ denotes $k$-th component of $u^{N}$. This yields

$$
\left\|\nabla\left(P(u)-P\left(u^{N}\right)\right)\right\|_{F_{p, q}^{s}} \leq C\left\|w^{N}\right\|_{F_{p, q}^{s}}\left(\left\|u^{N}\right\|_{F_{p, q}^{s}}+\|u\|_{F_{p, q}^{s}}\right) .
$$

Moreover, on account of Propositions 2.10-2.11, we find

$$
\left\|2^{j s}\left[u, \Delta_{j}\right] \cdot \nabla w^{N}\right\|_{L_{x}^{p} l_{j}^{q}} \leq C\left\|w^{N}\right\|_{F_{p, q}^{s}}\|u\|_{F_{p, q}^{s}} .
$$

Thereby the estimates (3.14), (3.15)-(3.16) in conjunction with (3.13) and (3.1) can imply

$$
\left\|w^{N}(t)\right\|_{F_{p, q}^{s}} \leq C\left\|w_{0}^{N}\right\|_{F_{p, q}^{s}}+C \int_{0}^{t}\left\|w^{N}(\tau)\right\|_{F_{p, q}^{s}} d \tau
$$

Applying Gronwall's inequality, we get

$$
\left\|w^{N}\right\|_{L_{T}^{\infty} F_{p, q}^{s}} \leq C\left\|w_{0}^{N}\right\|_{F_{p, q}^{s}} .
$$

from which (3.10) follows.

Step 4. Based on the aforementioned estimates, we show the continuity of the solution map. Let $\psi, u_{0} \in D(R)$, then

$$
\begin{aligned}
& \left\|S_{T}\left(u_{0}\right)-S_{T}(\psi)\right\|_{L_{T}^{\infty} F_{p, q}^{s}} \\
& \leq\left\|S_{T}\left(u_{0}\right)-S_{T}\left(P_{\leq N} u_{0}\right)\right\|_{L_{T}^{\infty} F_{p, q}^{s}}+\left\|S_{T}(\psi)-S_{T}\left(P_{\leq N} \psi\right)\right\|_{L_{T}^{\infty} F_{p, q}^{s}} \\
& +\left\|S_{T}\left(P_{\leq N} u_{0}\right)-S_{T}\left(P_{\leq N} \psi\right)\right\|_{L_{T}^{\infty} F_{p, q}^{s}} \\
& \leq C\left(\left\|u_{0}-P_{\leq N} u_{0}\right\|_{F_{p, q}^{s}}+\left\|\psi-P_{\leq N} \psi\right\|_{F_{p, q}^{s}}\right) \\
& +C\left\|S_{T}\left(P_{\leq N} u_{0}\right)-S_{T}\left(P_{\leq N} \psi\right)\right\|_{L_{T}^{\infty} F_{p, q}^{s-1}}^{\frac{1}{2}}\left\|S_{T}\left(P_{\leq N} u_{0}\right)-S_{T}\left(P_{\leq N} \psi\right)\right\|_{L_{T}^{\infty} F_{p, q}^{s+1}}^{\frac{1}{2}} \\
& \leq C\left(\left\|u_{0}-P_{\leq N} u_{0}\right\|_{F_{p, q}^{s}}+\left\|\psi-u_{0}\right\|_{F_{p, q}^{s}}\right)+C 2^{N / 2} R^{1 / 2}\left\|u_{0}-\psi\right\|_{F_{p, q}^{s-1}}^{\frac{1}{2}} .
\end{aligned}
$$

here we employed (3.3) and (3.12) in the last inequality. As $1 \leq p, q<\infty$, so for arbitrary $\epsilon>0$, one can select $N$ to be sufficiently large, such that

$$
C\left\|u_{0}-P_{\leq N} u_{0}\right\|_{F_{p, q}^{s}} \leq \frac{\epsilon}{2}
$$

Then fix $N$, choose $\delta$ so small that $\left\|u_{0}-\psi\right\|_{F_{p, q}^{s}}<\delta$ and $C \delta+C R^{1 / 2} 2^{N / 2} \delta^{1 / 2}<\epsilon / 2$. Hence,

$$
\left\|S_{T}\left(u_{0}\right)-S_{T}(\psi)\right\|_{L_{T}^{\infty} F_{p, q}^{s}}<\epsilon
$$

this concluded the proof. 


\section{Appendix A. Local Cauchy theory for the Euler equations}

In this appendix, we state and briefly show the well-known local in time existence and uniqueness for the Euler equations in Triebel-Lizorkin spaces $F_{p, q}^{s}\left(\mathbb{R}^{d}\right)$. For a completed treatment, one can refer to $[6,7]$. Recall that $D(R):=\left\{\phi \in F_{p, q}^{s}\right.$ : $\left.\|\phi\|_{F_{p, q}^{s}} \leq R, \operatorname{div} \phi=0\right\}$, the primary result of this part is as follows:

Theorem A.1. Let the space dimension $d \geq 2$ and $(s, p, q)$ be such that

$$
s>1+\frac{d}{p},(p, q) \in(1, \infty) \times(1, \infty) \quad \text { or } s \geq d+1, p=1, q \in[1, \infty) .
$$

Suppose $u_{0} \in D(R)$, then there exists some time $T=T(R)>0$ and a unique solution $u \in C\left([0, T] ; F_{p, q}^{s}\right)$ to the Euler equations.

Proof. As stated in Section 1, we will briefly outline the proof, as to the estimates involved, we omit the reasoning arguments and just present the result, which can essentially be established by applying Propositions (2.8)-(2.9) and Propositions (2.10)(2.11), see also Section 3. Let $\left(u^{(m)}, p^{(m)}\right)_{m \geq 0}$ be a sequence satisfying

$$
\left\{\begin{array}{l}
\partial_{t} u^{(m)}+u^{(m-1)} \cdot \nabla u^{(m)}+\nabla p^{(m-1)}=0 \\
\operatorname{div} u^{(m)}=0 \\
u^{(m)}(x, 0)=P_{\leq m} u_{0}
\end{array}\right.
$$

with $u^{(0)}=p^{(0)}=0,\left\|u_{0}\right\|_{F_{p, q}^{s}} \leq R$. The proof can be divided into five steps:

Step 1. First we claim that $u^{(m)}$ is uniformly bounded for some small time. Following argument that leads to (3.17), one can assert

$$
\left\|u^{(m)}(t)\right\|_{F_{p, q}^{s}} \leq\left\|P_{\leq m} u_{0}\right\|_{F_{p, q}^{s}}+C \int_{0}^{t}\left\|u^{(m-1)}(\tau)\right\|_{F_{p, q}^{s}}\left\|u^{(m)}(\tau)\right\|_{F_{p, q}^{s}} d \tau .
$$

Thus by Remark 4,

$$
\left\|u^{(m)}\right\|_{L_{T}^{\infty} F_{p, q}^{s}} \leq C\left\|u_{0}\right\|_{F_{p, q}^{s}}+C T\left\|u^{(m-1)}\right\|_{L_{T}^{\infty} F_{p, q}^{s}}\left\|u^{(m)}\right\|_{L_{T}^{\infty} F_{p, q}^{s}} .
$$

Now we specify $T \leq \tilde{T}_{0}$ by taking $8 C^{2} \tilde{T}_{0}\left\|u_{0}\right\|_{F_{p, q}^{s}} \leq 1$, then it follows by standard induction argument that

$$
\left\|u^{(m)}\right\|_{L_{\widetilde{T}_{0}}^{\infty} F_{p, q}^{s}} \leq 2 C\left\|u_{0}\right\|_{F_{p, q}^{s}}, \quad \forall m \geq 0 .
$$

Moreover

$$
\begin{aligned}
\left\|u^{(m)}\right\|_{L_{T}^{\infty} F_{p, q}^{s+1}} \leq C\left\|P_{\leq m} u_{0}\right\|_{F_{p, q}^{s+1}}+C T\left\|u^{(m-1)}\right\|_{L_{T}^{\infty} F_{p, q}^{s}}\left\|u^{(m)}\right\|_{L_{T}^{\infty} F_{p, q}^{s+1}} \\
+C T\left\|u^{(m-1)}\right\|_{L_{T}^{\infty} F_{p, q}^{s+1}}\left\|u^{(m)}\right\|_{L_{T}^{\infty} F_{p, q}^{s}} \\
\leq C 2^{m}\left\|u_{0}\right\|_{F_{p, q}^{s}}+C T R\left(\left\|u^{(m-1)}\right\|_{L_{T}^{\infty} F_{p, q}^{s+1}}+\left\|u^{(m)}\right\|_{L_{T}^{\infty} F_{p, q}^{s+1}}\right) .
\end{aligned}
$$

Iterating again, one can find some $T_{0}=T_{0}(R) \leq \tilde{T}_{0}$, such that

$$
\left\|u^{(m)}\right\|_{L_{T_{0}}^{\infty} F_{p, q}^{s+1}} \leq 4 C 2^{m}\left\|u_{0}\right\|_{F_{p, q}^{s}} .
$$

Since $u^{(m)}$ also solves the following integral equation(Duhamel formula)

$$
u^{(m)}(x, t)=P_{\leq m} u_{0}+\int_{0}^{t} \mathbb{P}\left(u^{(m-1)} \cdot \nabla u^{(m)}\right)(\tau) d \tau .
$$


where $\mathbb{P}:=\mathrm{Id}-\nabla \Delta^{-1}$ div is the Leray projector operator onto divergence free vector field. We readily see

$$
\begin{aligned}
& \left\|u^{(m)}\left(t_{1}\right)-u^{(m)}\left(t_{2}\right)\right\|_{F_{p, q}^{s}} \\
& \leq\left|\int_{t_{1}}^{t_{2}}\left\|\mathbb{P}\left(u^{(m-1)} \cdot \nabla u^{(m)}\right)\right\|_{F_{p, q}^{s}} d \tau\right| \\
& \leq C\left|\int_{t_{1}}^{t_{2}}\left\|u^{(m-1)}\right\|_{F_{p, q}^{s}}\left\|u^{(m)}\right\|_{F_{p, q}^{s}}+\left\|u^{(m-1)}\right\|_{F_{p, q}^{s-1}}\left\|u^{(m)}\right\|_{F_{p, q}^{s+q}} d \tau\right| \\
& \leq C 2^{m} R^{2}\left|t_{1}-t_{2}\right| .
\end{aligned}
$$

where we used (A.2) and (A.3) in the last step. This infers that for each fixed $m \geq 0$, $u^{(m)} \in C\left(\left[0, T_{0}\right] ; F_{p, q}^{s}\right)$.

Step 2. Let $v_{0} \in D(c R)$ for some universal constant $c,\left\{v^{(m)}, q^{(m)}\right\}_{m \geq 0}$ solves (A.1) with initial data $P_{\leq m} v_{0}$, we claim that there exist some $T_{1}=T_{1}(R)$ and a constant $C$ independent of $m$, such that

$$
\left\|u^{(m)}-v^{(m)}\right\|_{L_{T_{1}}^{\infty} F_{p, q}^{s-1}} \leq C\left\|u_{0}-v_{0}\right\|_{F_{p, q}^{s-1}}, \quad \forall m \geq 0 .
$$

Indeed, according to results in Step 1, one can say there exists some $\tilde{T}_{1}=\tilde{T}_{1}(R)$, s.t.

$$
\left\|u^{(m)}\right\|_{L_{\tilde{T}_{1}}^{\infty} F_{p, q}^{s}} \leq C\left\|u_{0}\right\|_{F_{p, q}^{s},}, \quad\left\|v^{(m)}\right\|_{L_{\tilde{T}_{1}}^{\infty} F_{p, q}^{s}} \leq C\left\|v_{0}\right\|_{F_{p, q}^{s}}, \quad \forall m \geq 0
$$

Now set $w^{(m)}=u^{(m)}-v^{(m)}, w_{0}=u_{0}-v_{0}$, then

$$
\left\{\begin{array}{l}
\partial_{t} w^{(m)}+u^{(m-1)} \cdot \nabla w^{(m)}+w^{(m-1)} \cdot \nabla v^{(m)}+\nabla\left(p^{(m-1)}-q^{(m-1)}\right)=0 \\
\operatorname{div} w^{(m)}=0 \\
w^{(m)}(x, 0)=w_{0}^{(m)}=P_{\leq m} w_{0}
\end{array}\right.
$$

Hence,

$$
\begin{aligned}
\left\|w^{(m)}(t)\right\|_{F_{p, q}^{s-1}} \leq\left\|w_{0}^{(m)}\right\|_{F_{p, q}^{s-1}} & +\int_{0}^{t}\left\|w^{(m-1)} \cdot \nabla v^{(m)}\right\|_{F_{p, q}^{s-1}}+\left\|\nabla\left(p^{(m-1)}-q^{(m-1)}\right)\right\|_{F_{p, q}^{s-1}} \\
& +\left\|2^{j(s-1)}\left[u^{(m-1)}, \Delta_{j}\right] \cdot \nabla w^{(m)}\right\|_{L_{x}^{p} l_{j}^{q}} d \tau \\
\leq\left\|w_{0}^{(m)}\right\|_{F_{p, q}^{s-1}} & +C \int_{0}^{t}\left\|w^{(m-1)}\right\|_{F_{p, q}^{s-1}}\left\|v^{(m)}\right\|_{F_{p, q}^{s}} \\
& +\left\|w^{(m)}\right\|_{F_{p, q}^{s-1}}\left\|u^{(m-1)}\right\|_{F_{p, q}^{s}} d \tau .
\end{aligned}
$$

Applying (A.5) with some $T_{1} \leq \tilde{T}_{1}$, we have

$$
\left\|w^{(m)}(t)\right\|_{L_{T_{1}}^{\infty} F_{p, q}^{s-1}} \leq C\left\|w_{0}\right\|_{F_{p, q}^{s-1}}+C T_{1} R\left(\left\|w^{(m-1)}\right\|_{L_{T_{1}}^{\infty} F_{p, q}^{s-1}}+\left\|w^{(m)}\right\|_{L_{T_{1}}^{\infty} F_{p, q}^{s-1}}\right)
$$

Selecting $T_{1}$ so small that $C T_{1} R \leq 1 / 8$, by an induction argument, one can immediately see

$$
\left\|w^{(m)}\right\|_{L_{T_{1}}^{\infty} F_{p, q}^{s-1}} \leq 2 C\left\|w_{0}\right\|_{F_{p, q}^{s-1}}, \quad \forall m \geq 0
$$

This yields (A.4). 
Step 3. Let $u^{(m, N)}$ solves equation (A.1) with initial data $P_{\leq m} P_{\leq N} u_{0}$, i.e.

$$
\left\{\begin{array}{l}
\partial_{t} u^{(m, N)}+u^{(m-1, N)} \cdot \nabla u^{(m, N)}+\nabla p^{(m-1, N)}=0 \\
\operatorname{div} u^{(m, N)}=0 \\
u^{(m, N)}(x, 0)=P_{\leq m} P_{\leq N} u_{0}
\end{array}\right.
$$

where $u^{(0, N)}=p^{(0, N)}=0$. Then there exist some $T_{2}=T_{2}(R)$ and a constant $C$ independent of $m, N$, satisfying

$$
\left\|u^{(m)}-u^{(m, N)}\right\|_{L_{T_{2}}^{\infty} F_{p, q}^{s}} \leq C\left\|u_{0}-P_{\leq N} u_{0}\right\|_{F_{p, q}^{s}} .
$$

We can argue as follows: by the estimates in Step $1, \exists \tilde{T}_{2}=\tilde{T}_{2}(R) \leq T_{0}$,

$$
\left\|u^{(m, N)}\right\|_{L_{\tilde{T}_{2}}^{\infty} F_{p, q}^{s+k}} \leq C 2^{N k}\left\|u_{0}\right\|_{F_{p, q}^{s}}, \quad \forall m \geq 0, k=0,1 .
$$

Now let $w^{(m, N)}=u^{(m)}-u^{(m, N)}, w_{0}^{(N)}:=u_{0}-P_{\leq N} u_{0}$, then

$$
\left\{\begin{array}{l}
\partial_{t} w^{(m, N)}+u^{(m-1)} \cdot \nabla w^{(m, N)}+w^{(m-1, N)} \cdot \nabla u^{(m, N)}+\nabla\left(p^{(m-1)}-p^{(m-1, N)}\right)=0, \\
\operatorname{div} w^{(m, N)}=0, \\
w^{(m, N)}(x, 0)=w_{0}^{(m, N)}=P_{\leq m} w_{0}^{(N)} .
\end{array}\right.
$$

Similarly, for $0<t \leq T_{2} \leq \tilde{T}_{2}$,

$$
\begin{aligned}
& \left\|w^{(m, N)}(t)\right\|_{F_{p, q}^{s}} \\
\leq & \left\|w_{0}^{(m, N)}\right\|_{F_{p, q}^{s}}+\int_{0}^{t} C 2^{N} R\left\|w^{(m-1, N)}\right\|_{F_{p, q}^{s-1}}+C R\left\|w^{(m, N)}\right\|_{F_{p, q}^{s}}+C R\left\|w^{(m-1, N)}\right\|_{F_{p, q}^{s}} d \tau .
\end{aligned}
$$

Noticing formula (A.4), we obtain

$$
\left\|w^{(m, N)}\right\|_{L_{T_{2}}^{\infty} F_{p, q}^{s}} \leq C\left\|w_{0}^{(N)}\right\|_{F_{p, q}^{s}}+C R T_{2}\left(\left\|w^{(m-1, N)}\right\|_{L_{T_{2}}^{\infty} F_{p, q}^{s}}+\left\|w^{(m, N)}\right\|_{L_{T_{2}}^{\infty} F_{p, q}^{s}}\right) .
$$

Now choosing $C T_{2} R$ small enough and performing an induction on $m$, we have

$$
\left\|w^{(m, N)}\right\|_{L_{T_{2}}^{\infty} F_{p, q}^{s}} \leq 2 C\left\|w_{0}^{(N)}\right\|_{F_{p, q}^{s}}, \quad \forall m, N \geq 0 .
$$

The desired result then follows.

Step 4. Next we show $\left\{u^{(m)}\right\}$ is a Cauchy sequence in $C\left(\left[0, T_{*}\right] ; F_{p, q}^{s-1}\right)$ for some $T_{*}=T_{*}(R) \leq \min \left\{T_{0}, T_{2}\right\}=\bar{T}$. In fact, set $w^{(m)}=u^{(m)}-u^{(m-1)}$, one can easily see $w^{(m+1)}$ satisfies

$$
\left\{\begin{array}{l}
\partial_{t} w^{(m+1)}+u^{(m)} \cdot \nabla w^{(m+1)}+w^{(m)} \cdot \nabla u^{(m)}+\nabla\left(p^{(m)}-p^{(m-1)}\right)=0, \\
\operatorname{div} w^{(m+1)}=0 \\
w^{m+1}(0, x)=\Delta_{m+1} u_{0}
\end{array}\right.
$$

Likewise,

$$
\left\|w^{(m+1)}(t)\right\|_{F_{p, q}^{s-1}} \leq\left\|\Delta_{m+1} u_{0}\right\|_{F_{p, q}^{s-1}}+C \int_{0}^{t}\left\|u^{(m)}\right\|_{F_{p, q}^{s}}\left(\left\|w^{(m)}\right\|_{F_{p, q}^{s-1}}+\left\|w^{(m+1)}\right\|_{F_{p, q}^{s-1}}\right) d \tau .
$$

By (A.2), we get

$$
\begin{aligned}
\left\|w^{(m+1)}\right\|_{L_{T_{*}}^{\infty} F_{p, q}^{s-1}} \leq C 2^{-(m+1)}\left\|u_{0}\right\|_{F_{p, q}^{s}} & +C T_{*} R\left\|w^{(m+1)}\right\|_{L_{T_{*}}^{\infty} F_{p, q}^{s-1}} \\
& +C T_{*} R\left\|w^{(m)}\right\|_{L_{T_{*}}^{\infty} F_{p, q}^{s-1}}
\end{aligned}
$$


Now choosing $T_{*} \leq \bar{T}$, such that $16 C^{2} T_{*} R \leq 1 / 2$, by a simple iteration, one can show

$$
\left\|w^{(m)}\right\|_{L_{T_{*}}^{\infty} F_{p, q}^{s-1}} \leq(4 C) 2^{-m}\left\|u_{0}\right\|_{F_{p, q}^{s},}, \quad \forall m \geq 0 .
$$

This exponential decay implies what we want.

Step 5. Finally we prove $\left\{u^{(m)}\right\}_{m \geq 0}$ is a Cauchy sequence in $X_{T_{*}}^{s}=C\left(\left[0, T_{*}\right] ; F_{p, q}^{s}\right)$. According to the conclusion in Step 4, one can also claim that $\left\{u^{(m, N)}\right\}_{m \geq 0}$ is a Cauchy sequence with

$$
\left\|u^{(m, N)}-u^{(n, N)}\right\|_{L_{T_{*}}^{\infty} F_{p, q}^{s-1}} \leq C 2^{-m} R, \quad n \geq m, \forall N \geq 0,
$$

here $C$ doesn't depend on $m, n, N$. As a consequence, for $n \geq m$,

$$
\begin{aligned}
& \left\|u^{(m)}-u^{(n)}\right\|_{L_{T_{*}}^{\infty} F_{p, q}^{s}} \\
& \leq\left\|u^{(m)}-u^{(m, N)}\right\|_{L_{T_{*}}^{\infty} F_{p, q}^{s}}+\left\|u^{(m, N)}-u^{(n, N)}\right\|_{L_{T_{*}}^{\infty} F_{p, q}^{s}}+\left\|u^{(n, N)}-u^{(n)}\right\|_{L_{T_{*}}^{\infty} F_{p, q}^{s}} \\
& \leq C\left\|u_{0}-P_{\leq N} u_{0}\right\|_{F_{p, q}^{s}}+C\left\|u^{(m, N)}-u^{(n, N)}\right\|_{L_{T_{*}}^{\infty} F_{p, q}^{s-1}}^{\frac{1}{2}}\left\|u^{(m, N)}-u^{(n, N)}\right\|_{L_{T_{*}}^{\infty} F_{p, q}^{s+1}}^{\frac{1}{2}} \\
& \leq C\left\|u_{0}-P_{\leq N} u_{0}\right\|_{F_{p, q}^{s}}+C 2^{-m / 2} 2^{N / 2} R .
\end{aligned}
$$

Since $1 \leq p, q<\infty$, the Schwartz function is dense in $F_{p, q}^{s}$, see [20], one can assert that the first term $C\left\|u_{0}-P_{\leq N} u_{0}\right\|_{F_{p, q}^{s}}$ can be made arbitrarily small provided that $N$ is large enough. Then fix such $N$, taking $m$ to be sufficiently large, the second term $C 2^{-m / 2} 2^{N / 2} R$ can also be as small as we want, so $\left\{u^{(m)}\right\}_{m \geq 0}$ is a Cauchy sequence in $X_{T_{*}}^{s}$ and converges to some $u \in X_{T_{*}}^{s}$. In view of (A.1), we find that the limit $u$ is a solution of the Euler system with initial data $u_{0} \in F_{p, q}^{s}$ and meets (A.2) as well. This finishes the local existence of solution in $X_{T_{*}}^{s}$, as to the uniqueness, which essentially can be done in the same way as the estimate in Step 4, we refer reader to [6] for more details.

Acknowledgements. Z. Guo is partially supported by ARC DP170101060.

\section{REFERENCES}

[1] H. Bahouri, J-Y. Chemin, And R. Danchin, Fourier analysis and nonlinear partial differential equations, Grundlehren der Mathematischen Wissenschaften 343, Springer, Heidelberg, 2011.

[2] J.-M. Bony, Calcul symbolique et propagation des singularités pour les équations aux dérivées partielles non linéaires, Ann. Sci. École Norm. Sup. (4) 14 (1981), pp. 209-246.

[3] J. Bourgain And D. LI, Strong ill-posedness of the incompressible Euler equation in borderline Sobolev spaces, Invent. Math. 201 (2015), pp. 97-157.

[4] J. Bourgain And D. LI, Strong illposedness of the incompressible Euler equation in integer $C^{m}$ spaces, Geom. Funct. Anal. 25 (2015), pp. 1-86.

[5] J. L. Bona And R. Smith, The initial-value problem for the Korteweg-de Vries equation, Philos. Trans. R. Soc. Lond. Ser. A, 278 (1975), pp. 555-601.

[6] D. Chat, On the well-posedness of the Euler equations in the Triebel-Lizorkin spaces, Comm. Pure Appl. Math. 55 (2002), pp. 654-678.

[7] D. Chat, On the Euler equations in the critical Triebel-Lizorkin spaces, Arch. Ration. Mech. Anal. 170 (2003), pp. 185-210.

[8] D. Chat, Local existence and blowup criterion for the Euler equations in the Besov spaces, Asymptot. Anal. 38 (2004), pp. 339-358.

[9] Q. Chen, C. Miao, And Z. Zhang, On the well-posedness of the ideal MHD equations in the Triebel-Lizorkin spaces, Arch. Ration. Mech. Anal. 195 (2010), pp. 561-578.

[10] C. Fefferman and E. M. Stein, Some maximal inequalities, Amer. J. Math. 93 (1971), pp. 107-115. 
[11] M. Frazier, R. Torres And G. Weiss, The boundedness of Calderón-Zygmund operators on the spaces $\dot{F}_{p}^{\alpha, q}$, Rev. Mat. Iberoamericana 4 (1988), pp. 41-72.

[12] Z. Guo, J. Li, AND Z. Yin, Local well-posedness of the incompressible Euler equations in $B_{\infty, 1}^{1}$ and the inviscid limit of the NavierStokes equations, J. Funct. Anal. (2018), https://doi.org/10.1016/j.jfa.2018.07.004

[13] A. Himonas and G. MisioŁeK, Non-uniform dependence on initial data of solutions to the Euler equations of hydrodynamics, Comm. Math. Phys. 296 (2010), pp. 285-301.

[14] B. Jawerth, Some observations on Besov and Lizorkin-Triebel Spaces, Math. Sacand. 40 (1977), pp. 94-104.

[15] T. KATO, Nonstationary flows of viscous and ideal fluids in $\mathbb{R}^{3}$, J. Funct. Anal. 9 (1972), pp. 296-305.

[16] T. Kato And G. Ponce, Commutator estimates and the Euler and Navier-Stokes equations, Comm. Pure Appl. Math. 41 (1988), pp. 891-907.

[17] H. C. PAK AND Y. J. PARK, Existence of solution for the Euler equations in a critical Besov space $B_{\infty, 1}^{1}\left(\mathbb{R}^{d}\right)$, Comm. Partial Differential Equations, 29 (2004), pp. 1149-1166.

[18] E. M. StEIn, Harmonic analysis: real-variable methods, orthogonality, and oscillatory integrals, Princeton Mathematical Series 43, Princeton University Press, Princeton, NJ, 1993.

[19] R. TAKADA, Counterexamples of commutator estimates in the Besov and the Triebel-Lizorkin spaces related to the Euler equations. SIAM J. Math. Anal. 42(6) (2010), pp. 2473-2483.

[20] H. TRIEBEL, Theory of function spaces, Birkhäuser, 1983.

[21] M. Vishik, Hydrodynamics in Besov spaces, Arch. Ration. Mech. Anal. 145 (1998), pp. 197214.

[22] M. VishiK, Incompressible flows of an ideal fluid with vorticity in borderline spaces of Besov type, Ann. Sci. École Norm. Sup.(4) 32 (1999), pp. 769-812.

[23] B. Wang, Z. Huo, C. Hao And Z. Guo, Harmonic Analysis Methods for Nonlinear Evolution Equations. I, World Scientific Publishing Co. Pte. Ltd., Hackensack, NJ, 2011.

School of Mathematical Sciences, Monash University, Clayton ViC 3800, AusTRALIA

E-mail address: zihua.guo@monash.edu

School of Mathematical Sciences, Monash University, Clayton ViC 3800, AusTRALIA

E-mail address: likjie@163.com 